\title{
A Note on the Role of Mean Flows in Doppler-Shifted Frequencies
}

\author{
Theo Gerkema, Leo R. M. MAas, ANd Hans van HaRen \\ NIOZ Royal Netherlands Institute for Sea Research, Texel, Netherlands
}

(Manuscript received 21 May 2012, in final form 28 September 2012)

\begin{abstract}
The purpose of this paper is to resolve a confusion that may arise from two quite distinct definitions of "Doppler shifts": both are used in the oceanographic literature but they are sometimes conflated. One refers to the difference in frequencies measured by two observers, one at a fixed position and one moving with the mean flow-here referred to as "quasi-Doppler shifts." The other definition is the one used in physics, where the frequency measured by an observer is compared to that of the source. In the latter sense, Doppler shifts occur only if the source and observer move with respect to each other; a steady mean flow alone cannot create a Doppler shift. This paper rehashes the classical theory to straighten out some misconceptions. It is also discussed how wave dispersion affects the classical relations and their application.
\end{abstract}

\section{Introduction}

A much studied situation in physical oceanography is linear wave propagation in the presence of a mean flow. Right at the outset, it is useful to identify the three key players in this problem: the wave source, the observer, and the mean flow. The source and observer may move relative to each other as well as relative to the mean flow. In the midnineteenth century, Christian Doppler identified the circumstances under which the frequency measured by an observer will be different from the frequency at which the source emits its waves. In physics, this difference is called the Doppler shift.

In the oceanographic literature, however, the term is often used in another sense, for example, in Bretherton and Garrett (1968), Olbers (1981), Kunze (1985), and Bühler (2009). Their starting point lies in the equations of motion, where a mean flow $U$ gives a combination of terms

$$
\frac{\partial}{\partial t}+U \frac{\partial}{\partial x}+\cdots
$$

For sinusoidal waves $\sin (\omega t-k x)$, with frequency $\omega$ and wavenumber $k$, this becomes $\omega-U k$. It is then common to define $\omega^{\prime}=\omega-U k$, the so-called "intrinsic frequency." Lighthill (1978) comments on the nomenclature of this expression:

Corresponding author address: Theo Gerkema, Royal NIOZ, P.O. Box 59, 1790 AB Den Burg, Texel, Netherlands.

E-mail: gerk@nioz.nl
"In the context of moving sources, equation $\left[\omega^{\prime}=\omega-\right.$ $U k]$ has long been called the Doppler relationship, and it is natural to call it by this name also for waves propagating through non-uniformly moving fluids" (p. 326, our italics).

Thus, it has become common to speak of $U k$ as a "Doppler shift," referring to the difference between the frequency $\omega$ measured by an observer at rest and the frequency $\omega^{\prime}$ measured by an observer moving with the mean flow. So, here the term is used to indicate the difference in frequency between two observers, in contrast to normal parlance in physics, where it refers to the difference in frequency between source and observer.

For the sake of clarity, we shall refer to the Doppler shift in the former, "Bretherton/Garrett" sense as quasiDoppler shift, reserving the term Doppler shift for its normal meaning in physics. The distinction is important, for an observer may experience a quasi-Doppler shift, while having no Doppler shift-and vice versa. A mean flow can be said to imply a quasi-Doppler shift, but a mean flow cannot create a Doppler shift.

Is this merely a matter of semantics? In the aforementioned references, sources were left out of the equation; the waves are simply assumed to be there. The fixed observer measures frequency $\omega$, while the observer moving with the flow measures $\omega^{\prime}$, and these frequencies can be compared (quasi-Doppler shift), but no comparison with the forcing frequency can be made because none is involved. So far, no confusion is possible. But, as soon as one applies these theories to a specific oceanographic situation, sources inevitably come into play-for 
example, the forcing of near-inertial waves by the wind or of internal tides by tidal flow over topography. Indeed, in observations it is more common to look into the relation between the measured frequency and the forcing frequency than it is to compare a frequency from a moored instrument with simultaneous measurements from a drifter. It is, therefore, crucial for the correct interpretation of oceanographic data to understand precisely under what circumstances a Doppler shift may, or may not, occur. However, the distinct meanings of quasi-Doppler shifts and Doppler shifts are sometimes conflated. It is, for example, not uncommon to see the notion of a quasi-Doppler shift being morphed into the (incorrect) idea that a mean flow would give rise to a Doppler-shifted frequency at a mooring. Some instances are discussed in section 4 .

The purpose of this paper is twofold: to bring into clear focus the distinction between quasi-Doppler shifts and Doppler shifts, and to provide a concise overview of the role of mean flows in Doppler shifts. Regarding the latter point, this paper has partly the character of a review; much of it was already known by the late nineteenth century, although these insights seem to have somewhat slipped into oblivion. An additional element is the inclusion of dispersion. The classical theory typically considers dispersionless waves (sound, light waves), but in the oceanographic context, dispersion is usually important.

We start by rehashing the basic theory of Doppler shifts (section 2) following its historical origins. This section also serves to stress the importance of specifying and distinguishing the movements of the source, medium, and observer. We then examine an analytical example in which not only the classical Doppler relation for the shift in frequency is derived, but also expressions for amplitude and wavelength (section 3). Until here, waves are assumed to be dispersionless. In section 4 , it is shown that the effects of dispersion can be straightforwardly accommodated in the classical relation for Doppler shifts. In section 5 we discuss some observations on internal waves from the literature and revisit their interpretation with regard to Doppler shifts. Conclusions follow in section 6; here we also discuss, briefly, the effect of nonsteady mean flows but, in preceding sections, steadiness is assumed.

\section{Recapitulation of the Doppler theory}

Of the several papers in which Doppler laid out the effect named after him, three are of particular interest here. They were published in 1842, 1846, and 1847 and later reprinted in Doppler (1907). This book is more useful than the original papers thanks to extensive notes by H. A. Lorentz, which contain numerous corrections and clarifications.

In his theory of 1842 , Doppler distinguished the two cases of a moving source and a moving observer. The medium is assumed to be at rest. Waves are emitted from the source at period $T_{s}$; they propagate away at phase speed $c$. An important restriction, here and in the following section, is that waves are assumed to be dispersionless. In other words, the proper phase speed $c$ is a constant and is independent of the wave period; it depends solely on the properties of the medium (e.g., on temperature in the case of sound waves).

The first case is one in which the observer is at rest, while the source moves. Let its speed be $v_{s}$, directed toward the observer. Each wave crest has to bridge the distance between the position of emittance and the position of the observer, but this distance decreases by an amount of $v_{s} T_{s}$ for every next wave crest because of the movement of the source. The period between the arrival of successive crests is therefore not $T_{s}$, but the Doppler shifted $T_{1}=T_{s}-v_{s} T_{s} / c=T_{s}\left(1-v_{s} / c\right)$. The wavelength being shifted as $\lambda_{1}=\left(c-v_{s}\right) T_{s}$, the relation $c=\lambda_{1} / T_{1}$ is satisfied from the viewpoint of the observer.

The second case is one in which the source is at rest while the observer moves. Suppose the observer moves away from the source at speed $v_{o}$. At a moment when a wave crest passes the observer, the following crest is still at a distance $\lambda=c T_{s}$, the wavelength. Approaching the observer at a relative speed of $c-v_{o}$, that following crest will arrive after a time interval $T_{2}=\lambda /\left(c-v_{o}\right)=$ $T_{s} /\left(1-v_{o} / c\right)$. This is now the Doppler shifted period. There is no change in wavelength in this case.

The first case was soon put to the test by the physicist (and later meteorologist) Buys Ballot. In 1845, on a newly built steam train near Utrecht, he had musicians play tones on a trumpet, with other musicians standing along the railway to register the pitch of the tones before and after the passage of the train. The change in pitch was found to be in good agreement with Doppler's formula. This was in itself a triumphant confirmation of his principle, but Doppler's chief interest lay in the application to the color of the stars, which proved to be less straightforward (for more on this history, see Toman 1984).

In his 1846 paper, Doppler considered the situation in which the source and observer are both in movement. Thus, combining the previous two cases, the observed period $T$ becomes

$$
T=T_{s} \frac{c-v_{s}}{c-v_{o}}
$$

For a proper application of this formula, positive values should be assigned to $v_{s}$ and $v_{o}$ for movement to the 


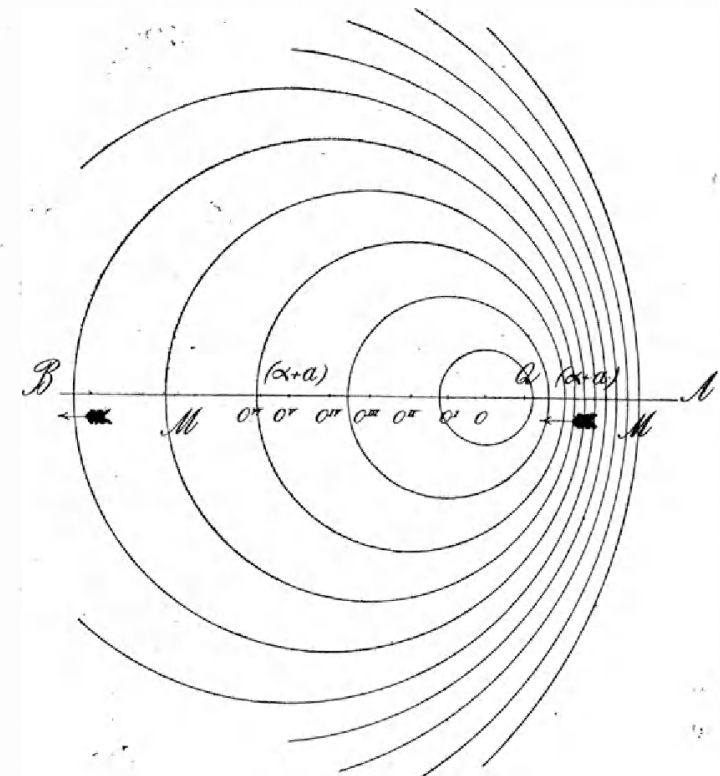

FiG. 1. Illustration from Doppler's 1847 paper (Doppler 1907), showing the effect of a medium $M$ flowing to the left (as indicated by the arrows). The source is at rest at $Q$, as are the observers at $A$ and $B$. The mean flow produces a leftward translation of the circular wave pattern; at this instant, previously generated wave crests have their centers at $O, O^{I}, O^{I I}$, etc. Here the mean flow is assumed to be lower than the proper phase speed of the waves (otherwise no waves would move to the right at all)

right; negative values, for movement to the left. However, we will take $c$ always positive. If the observer is to the left of the source, we are concerned with waves moving to the left and, accordingly, should replace $c$ by $-c$ in the formula.

Expression (2) has an obvious but important corollary: if the source and observer move at the same speed and in the same direction (i.e., $v_{s}=v_{o}$ ), no Doppler shift in frequency occurs. But then, in a frame of reference that moves together with the source and observer, the medium will be perceived to be moving. One thus arrives immediately at the conclusion that a moving medium creates no Doppler shift in frequency.

This brings us to the case considered by Doppler in his 1847 paper, in which he studied the effect of a moving medium on waves in ether (i.e., light waves), air, or water. The source and observer are here assumed to be at rest. For an observer located upstream ( $A$ in Fig. 1), the waves travel at a lower speed and the wavelength is shortened, while for an observer located downstream $(B)$ the speed and wavelength are enlarged. But, what happens to the frequency? For an observer at any fixed position, successive wave crests propagate under identical circumstances from source to observer and hence need equal times to bridge that distance; as a consequence, the observed frequency must be the same as the frequency of emittance. This remarkably simple argument, given by Lorentz, hangs on the stationarity of the setting and remains valid if the mean flow is spatially nonuniform.

By the late nineteenth century, this result had already found its way into the textbooks, for example, Lord Rayleigh (1896, p.154):

"If the source and the observer move with the same velocity there is no alteration of frequency, whether the medium be in motion, or not."

The only way the observed frequency can be higher (or lower) is when the relation between source and observer is nonstationary: if they move toward (or away from) each other. In that case, successive waves do not propagate under identical circumstances, for the distance between source and observer, which they have to bridge, changes.

The different situations, discussed here, are elegantly captured in a single formula derived by Bateman (1917):

$$
T=T_{s} \frac{c+U-v_{s}}{c+U-v_{o}} .
$$

Again, we adopt the convention with regard to the sign of $c$ as in (2).

Bateman's Eq. (3) is a natural generalization of (2) in that the proper phase speed $c$ in the latter is being replaced by the effective phase speed $c+U$. Equation (3) confirms that a mean flow $U$ does not create a Doppler shift; a mean flow merely modifies an already existing Doppler shift if the source and observer are in relative movement (i.e., $v_{s} \neq v_{o}$ ). This formula will be rederived in the next section, where we explore an analytical example.

\section{An analytical example}

We will examine the propagation of linear long interfacial waves in the presence of a steady and uniform background flow $U$. This simple example allows us to pinpoint, by means of an explicit solution, the distinct roles of the movement of the source, medium and observer.

\section{a. Basic equations}

We assume wave propagation in the horizontal $x$ direction in a two-layer system; for the other horizontal direction: $\partial / \partial y=0$. Within each layer the horizontal current velocity associated with the (long) waves is independent of the vertical; these velocities are called $u_{1}$ and $u_{2}$, for upper and lower layer, respectively. Then 
$u=u_{2}-u_{1}$, the change in horizontal velocity across the interface, satisfies the momentum equation

$$
u_{t}+U u_{x}=-g_{*} \eta_{x}
$$

with $g_{*}$ denoting reduced gravity; $\eta$ is the interfacial displacement. We ignore the Coriolis force here, but will discuss its dispersive effect in section 4 .

From the continuity equation and the boundary conditions (rigid lid at surface, flat bottom), one obtains

$$
\eta_{t}+U \eta_{x}+h_{*} u_{x}=F(t, x)
$$

Here $h_{*}$ is the reduced water depth $\left(h_{*}=h_{1} h_{2} /\left(h_{1}+h_{2}\right)\right.$, with $h_{i}$ the unperturbed thickness of layer $i$ ).

In (5), an ad hoc source is introduced through the forcing term $F$. For later use, we specify the source as a wavemaker oscillating at frequency $\omega_{s}$ and moving at speed $v_{s}$ :

$$
F(t, x)=\sin \left(\omega_{s} t\right) G^{\prime}\left(x-v_{s} t\right)
$$

For positive (negative) $v_{s}$, the source moves to the right (left). Here, $G^{\prime}$ is the derivative, with respect to its argument, of an arbitrary function $G$. This way of writing turns out to be convenient in what follows.

\section{b. Solution}

To facilitate solving (4) and (5) we make a transformation to a coordinate system moving along with the flow:

$$
\tilde{x}=x-U t ; \quad \tilde{u}(t, \tilde{x})=u(t, \tilde{x}+U t)
$$

and similarly for $\eta$ and $F$. Hence, by applying the chain rule

$$
u_{t}=\tilde{u}_{t}-U \tilde{u}_{\tilde{x}} ; \quad u_{x}=\tilde{u}_{\tilde{x}}
$$

and similarly for $\eta$. Transformed to this system, (4) and (5) become

$$
\begin{aligned}
\tilde{u}_{t} & =-g_{*} \hat{\eta}_{\tilde{x}} \\
\tilde{\eta}_{t}+h_{*} \tilde{u}_{\tilde{x}} & =\tilde{F}(t, \tilde{x}) .
\end{aligned}
$$

They can be combined into one equation for $\eta$ :

$$
\tilde{\eta}_{t t}-c^{2} \tilde{\eta}_{\tilde{x} \tilde{x}}=\tilde{F}_{t}
$$

with $c=\left(g_{*} h_{*}\right)^{1 / 2}$, the phase speed of wave propagation in the absence of a background flow. Like in the previous section, we define $c$ to be positive (unlike $U$ and $v_{s}$ ). For initial conditions $\tilde{\eta}, \tilde{\eta}_{t}=0$, starting from a system at rest, the solution of (8) reads (see, e.g., Zauderer 1989, §4.5):

$$
\tilde{\eta}(t, \tilde{x})=\frac{1}{2 c} \int_{0}^{t} d \tau \int_{\tilde{x}-c(t-\tau)}^{\tilde{x}+c(t-\tau)} d \xi \tilde{F}_{\tau}(\tau, \xi) .
$$

From (6) we obtain, after transformation to the moving frame of reference and taking the derivative to $\tau$,

$$
\begin{aligned}
\tilde{F}_{\tau}(\tau, \xi)= & \omega_{s} \cos \left(\omega_{s} \tau\right) G^{\prime}\left(\xi+\left(U-v_{s}\right) \tau\right) \\
& +\left(U-v_{s}\right) \sin \left(\omega_{s} \tau\right) G^{\prime \prime}\left(\xi+\left(U-v_{s}\right) \tau\right)
\end{aligned}
$$

The inner integral of (9) now yields, after some rewriting,

$$
\begin{aligned}
\int_{\tilde{x}-c(t-\tau)}^{\tilde{x}+c(t-\tau)} d \xi \tilde{F}_{\tau}= & \frac{\partial}{\partial \tau}\left\{\sin \left(\omega_{s} \tau\right)\left[G\left(\tilde{X}_{-}\right)-G\left(\tilde{X}_{+}\right)\right]\right\} \\
& +c \sin \left(\omega_{s} \tau\right)\left\{G^{\prime}\left(\tilde{X}_{-}\right)+G^{\prime}\left(\tilde{X}_{+}\right)\right\},
\end{aligned}
$$

with $\tilde{X}_{ \pm}=\tilde{x}-( \pm c) t+\left( \pm c+U-v_{s}\right) \tau$. Taking the integral $\int_{0}^{t} d \tau \cdots$, the first term on the rhs of $(10)$ vanishes entirely, so (9) becomes

$$
\tilde{\eta}(t, \tilde{x})=\frac{1}{2} \int_{0}^{t} d \tau \sin \left(\omega_{s} \tau\right)\left\{G^{\prime}\left(\tilde{X}_{-}\right)+G^{\prime}\left(\tilde{X}_{+}\right)\right\} .
$$

Transforming back to the original frame of reference $(\tilde{x}=x-U t)$, we get the final result:

$\eta(t, x)=\frac{1}{2} \int_{0}^{t} d \tau \sin \left(\omega_{s} \tau\right)\left\{G^{\prime}\left(X_{-}\right)+G^{\prime}\left(X_{+}\right)\right\}$,

with $X_{ \pm}=x-( \pm c+U) t+\left( \pm c+U-v_{s}\right) \tau$.

The remaining integral in (11) can be solved analytically if we choose a (moving) point source, described by a delta distribution: $G^{\prime}=\delta$. From the general properties of delta distributions (see, e.g., Messiah 1961), one has

$$
\delta\left(X_{ \pm}\right)=\delta\left(\left( \pm c+U-v_{s}\right)\left(\tau-\tau_{ \pm}^{*}\right)\right)=\frac{\delta\left(\tau-\tau_{ \pm}^{*}\right)}{\left| \pm c+U-v_{s}\right|},
$$

with $\tau_{ \pm}^{*}=[( \pm c+U) t-x] /\left( \pm c+U-v_{s}\right)$. The deltadistribution $\delta\left(X_{ \pm}\right)$yields a contribution if $0<\tau_{ \pm}^{*}<t$, and is zero otherwise. Thus, from (11) we can write the solution as

$$
\eta=\eta_{-}+\eta_{+}
$$

with

$$
\eta_{ \pm}(t, x)=\frac{\sin \left(\omega_{s} \tau_{\stackrel{*}{*}}\right)}{2\left| \pm c+U-v_{s}\right|} \quad \text { if } \quad 0<\tau \text { 类 }<t
$$

and $\eta_{ \pm}=0$ for all other $\tau_{ \pm}^{*}$. In the absence of mean flows, the terms $\eta_{-}$and $\eta_{+}$in (12) describe waves propagating 
observer 1

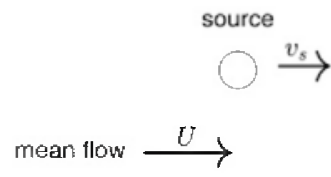

Flg. 2. Sketch of a situation used to illustrate the implications of (13). A source, initially located halfway between two fixed observers, may move to the right. The presence of a rightward mean flow is also considered.

to the left and right, respectively. Using the definition of $\tau_{ \pm}^{*}$, we can write $\eta_{+}$more simply as

$\eta_{ \pm}(t, x)=\frac{1}{2\left| \pm c+U-v_{s}\right|} \sin \omega_{s}\left(\frac{( \pm c+U) t-x}{ \pm c+U-v_{s}}\right)$

for all $x$ satisfying either $( \pm c+U) t<x<v_{s} t$ or $v_{s} t<x<$ $( \pm c+U) t$; for all other $x, \eta_{ \pm}=0$.

\section{c. Results}

We first consider the case of two fixed observers, as illustrated in Fig. 2. One is located to the left of the initial location of the source at a distance of two wavelengths; the other to the right of the source, also at two wavelengths. (Wavelength is here defined in the absence of a mean flow and with a source at rest.) The signal received by the observers, $\eta$, is calculated from (12), (13). In Fig. 3 they are plotted in gray and black, respectively. Wave forcing starts at $t=0$. Figure 3 a shows the reference case, which has no mean flow and no movement of the source; as expected, both observers start to receive the same signal after two forcing periods.

In Figs. 3b,c,d, we add a background mean flow $U$, but the source still does not move. Clearly, the observed wave period is unchanged in each of these cases; this confirms the classical result, discussed in the previous section, that a background flow does not create a Doppler shift in frequency. In Fig. 3b, the effective phase speed becomes $3 c / 2$ for rightward propagating waves, leading to an earlier arrival at the observer's position and a reduction in amplitude (black line). For leftward propagating waves, the background flow reduces the effective phase speed to $c / 2$, leading to a later arrival and an enhanced amplitude (gray line). In Figs. $3 c$, d the flow becomes too strong for the waves to propagate leftward, and the observer on the left detects no waves at all. In Fig. 3d, they are even drifted to the right so that the observer on the right starts to receive them after four wave periods.

In Figs. 3e,f we have no background flow; instead, the source moves to the right. Now we see, as expected, a Doppler shift in frequency. In Fig. 3e, we see the source passing the observer on the right at $t=4$; at that a) $\mathrm{U}=0, \mathrm{v}_{\mathrm{s}}=0$

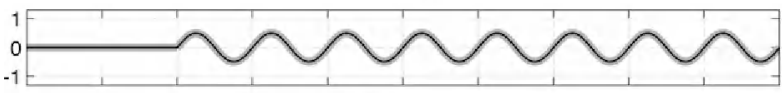

b) $\mathrm{U}=0.5 \mathrm{c}, \mathrm{v}_{\mathrm{s}}=0$

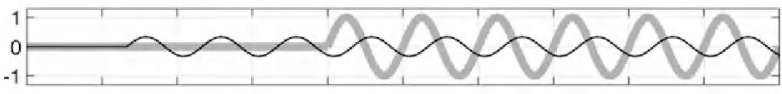

c) $U=C, v_{s}=0$

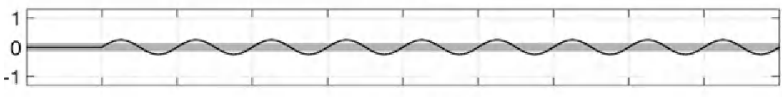

d) $\mathrm{U}=1.5 \mathrm{c}, \mathrm{v}_{\mathrm{s}}=0$

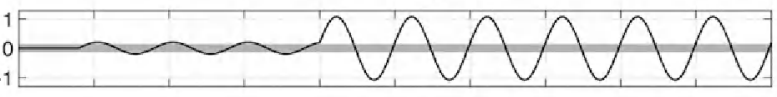

e) $U=0, v_{s}=0.5 \mathrm{c}$
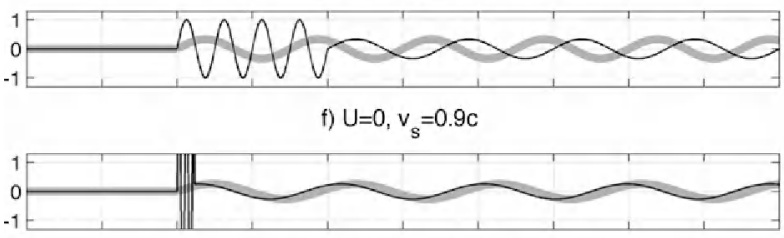

g) $U=0.5 c, v_{s}=0.5 c$

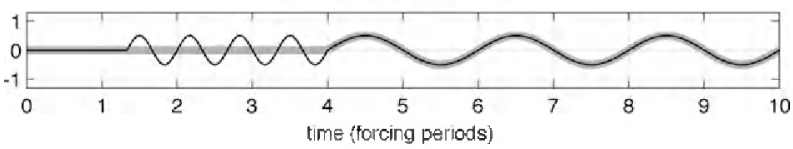

FIG. 3. Solutions of interfacial displacement $\eta$, defined by (12) and (13) for various $U$ and $v_{s}$, showing the received signal for an observer two wavelengths to the left of the (initial) position of the source (in gray), and another observer two wavelengths to the right (in black).

instant, the wave frequency drops sharply. From then on, both observers detect the same signal in terms of amplitude and (Doppler-shifted) wave period. The nearcritical case is shown in Fig. 3f, where we see a "boom" when the source passes the observer on the right (the peak is cut off in the figure, its amplitude is, in fact, nearly four times larger than shown).

The combined effect of a moving source and background flow in shown in Fig. 3g; the Doppler shift due to the moving source is now modified by the background flow (cf. Fig. 3e).

\section{d. Discussion}

We can readily adjust (13) to the case in which the observer moves as well, by the substitution $x=x_{o}+v_{o} t$. The argument of the sine in (13) then becomes

$$
\frac{ \pm c+U-v_{o}}{ \pm c+U-v_{s}} \omega_{s} t-\frac{\omega_{s}}{ \pm c+U-v_{s}} x_{o} .
$$

This expression neatly sums up all the possibilities. The first term in (14) gives the Doppler shift in frequency: 


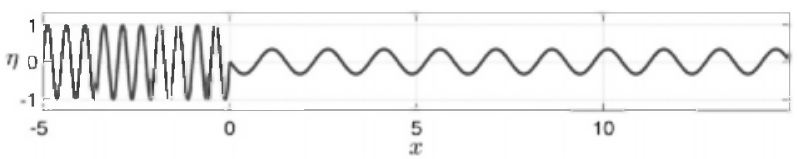

FIG. 4. Solution (13) after 10 forcing periods with the source at rest at $x=0$ and in the presence of a background flow $U=c / 2$. This case corresponds to the case of Fig. $3 \mathrm{~b}$. On the horizontal axis, $x$ has been scaled by the wavelength $\lambda=c T$, being the unperturbed wavelength in the absence of a background flow.

$$
\omega=\frac{ \pm c+U-v_{o}}{ \pm c+U-v_{s}} \omega_{s} .
$$

The movement of the observer $\left(v_{o}\right)$ implies a Doppler shift in frequency, unless it moves at the same velocity as the source $\left(v_{o}=v_{s}\right)$. A mean flow $U$ modifies the Doppler shift if the source and observer have a relative movement, but cannot by itself create a Doppler shift. The expression (15) confirms exactly the classical result (3) by Bateman (1917).

From the second term in (14) we see that the wavenumber is modified by a movement of the source and by a mean flow:

$$
k=\frac{\omega_{s}}{ \pm c+U-v_{s}} .
$$

This, too, is fully in agreement with the classical results (see section 2). An example is shown in Fig. 4, illustrating the effect of a mean flow on downstream and upstream propagating waves.

The analysis of Section $3 \mathrm{~b}$ yields, as an extra result, the amplitude factor $1 / \mid \pm c+U-v_{s}$ in (13), demonstrating how the amplitude is influenced by the mean flow (see also Fig. 4) and by the movement of the source.

\section{e. Quasi-Doppler shifts}

Returning now to the quasi-Doppler shift (as we call it), which is the concept often used in the oceanographic literature, as discussed in the introduction. In the quasiDoppler shift, $U k$ is the difference between two $a b$ served frequencies, one by a fixed observer and one by an observer moving along with the mean flow. We can now easily verify this relation. According to (15), a fixed observer $\left(v_{o}=0\right)$ measures frequency

$$
\omega=\frac{ \pm c+U}{ \pm c+U-v_{s}} \omega_{s} .
$$

An observer moving along with the mean flow $\left(v_{o}=U\right)$, meanwhile, measures frequency

$$
\omega^{\prime}=\frac{ \pm c}{ \pm c+U-v_{s}} \omega_{s} .
$$

Notice that both are affected by the movement of the source and, whenever source and observer are in relative movement, by the mean flow as well. Wavenumber $k$, given by (16), is also affected by the mean flow and the movement of the source. But together, they form the simple and familiar relation

$$
\omega^{\prime}=\omega-U k \quad \text { (quasi-Doppler shift) }
$$

which is valid whatever the value of $v_{s}$. Precisely because of this invariance for $\boldsymbol{v}_{s}$, however, the relation hides the fact that $\omega^{\prime}, \omega$, and $k$ are, each individually, affected by the movement of the source. To unveil this dependence, one has to examine the Doppler shift (sensu stricto), as above.

\section{Dispersive waves}

So far, we have considered dispersionless waves; the phase speed was given by a constant $c$, which is independent of the wave period. It depends only on properties of the medium; for example, in the previous section, it was given by $c=\left(g_{*} h_{*}\right)^{1 / 2}$.

If we consider a moving source (speed $v_{s}$ toward an observer at rest) emitting dispersive waves at forcing period $T_{s}$, we can again use the reasoning of the third paragraph of section 2 , but with one important modification: the emitted waves now travel at some (as yet unknown) speed $C$. Moreover, a distinction has to be made between the waves traveling ahead and behind the source, for their phase speeds will be different.

In all other respects, the earlier reasoning is valid regardless of whether the waves are dispersive or not: each wave crest has to bridge the distance between the position of emittance and the position of the observer, but this distance decreases by an amount of $v_{s} T_{s}$ for every next wave crest because of the movement of the source. The period between the arrival of successive crests is therefore not $T_{s}$, but the Doppler shifted

$$
T=T_{s} \frac{C-v_{s}}{C}
$$

This equation, however, is not immediately useful because it involves an unknown $T$ as well as an unknown $C$, which itself depends on the Doppler-shifted $T$. A dispersion relation is needed to close the problem.

As an example, we will introduce dispersion due to the Coriolis force (parameter $f$ ), known from Poincaré waves. We also include a steady uniform background flow $U$ : 


$$
\omega=U k+\left(f^{2}+c^{2} k^{2}\right)^{1 / 2} .
$$

With $\omega=2 \pi / T$ and the definition of phase speed $C=\omega / k$, we can solve $C$ from (18) and (19):

$C_{ \pm}=\frac{-\left(v_{s} f^{2}-U \omega_{s}^{2}\right) \pm \omega_{s}\left[c^{2}\left(\omega_{s}^{2}-f^{2}\right)+\left(U-v_{s}\right)^{2} f^{2}\right]^{1 / 2}}{\omega_{s}^{2}-f^{2}}$.

Thus, the two roots of the phase speed $C$ are expressed in terms of known parameters. With this, the Dopplershifted period $T$ can now be calculated from (18). Equation (20) demonstrates that the phase speed of dispersive waves is not only affected by a mean flow but also by the movement of the source.

Finally, a movement of the observer does not in any way change the characteristics of the wave itself (in particular, it leaves $C$ unaffected). So, the reasoning of section 2 dealing with a moving observer can be applied regardless of dispersion. The upshot is a generalization of Bateman's formula:

$$
T=T_{s} \frac{C_{ \pm}-v_{s}}{C_{ \pm}-v_{o}} .
$$

Here $C_{ \pm}$is again given by (20), following from (18) [not from (21)!] and the dispersion relation (19). The mean flow enters (21) via $C$ but, as before, does not by itself lead to any Doppler shift.

Notice that for $f=0,(20)$ would lead back to $C_{ \pm}=$ $\pm c+U$, the effective phase speed in the dispersionless case, reducing (21) to (3).

An alternative approach to examining the effects of dispersion would be to redo the analysis of section 3 with dispersion included. To some extent, this is feasible, but the problem can no longer be fully solved analytically, although a closed-form solution can be obtained.

For example, if we add Coriolis terms to (4) and (5), we get as a starting point, with an additional equation for the transverse velocity component $v$ :

$$
\begin{aligned}
u_{t}+U u_{x}-f v & =-g_{*} \eta_{x} \\
v_{t}+U v_{x}+f u & =0 \\
\eta_{t}+U \eta_{x}+h_{*} u_{x} & =F(t, x) .
\end{aligned}
$$

After a transformation to a system moving with the mean flow, this set can be reduced to one equation, for example, for $u$ :

$$
\tilde{u}_{t t}-c^{2} \tilde{u}_{\tilde{x} \tilde{x}}+f^{2} \tilde{u}=-g_{*} \tilde{F}_{\tilde{x}}(t, \tilde{x}),
$$

a forced Klein-Gordon equation. As before, $c$ is the constant $c=\left(g_{*} h_{*}\right)^{1 / 2}$. Equation (22) can be solved in terms of a double integral involving the Bessel function $J_{0}$ (Zauderer 1989). Choosing again a moving point source, one of these integrals can be solved. After a transformation to the original coordinates, the final expression reads

$$
\begin{aligned}
u(t, x)= & \frac{1}{2 c} \int_{0}^{t} d \tau \sin \omega \tau \\
& \times J_{0}\left\{\frac{f}{c} \sqrt{c^{2}(t-\tau)^{2}-\left[x-U t+\left(U-v_{s}\right) \tau\right]^{2}}\right\} \\
& \times \Theta\left\{c^{2}(t-\tau)^{2}-\left[x-U t+\left(U-v_{s}\right) \tau\right]^{2}\right\}
\end{aligned}
$$

Here $\Theta$ is the Heaviside stepfunction (equal to one for positive argument, zero elsewhere). The integral in (23) is not amenable to further analytical treatment, but it can be easily solved numerically. For various values of $v_{s}, U$, and $f$ we have checked whether the wave period found numerically is the same as the one obtained from the procedure (18) and (20). In all cases, they were found to be in agreement to within numerical accuracy, confirming the correctness of the above procedure.

\section{Observations and their interpretation}

The conflation of quasi-Doppler shifts and Doppler shifts has sometimes featured in interpretations of moored observations on internal waves (e.g., Frankignoul 1970; White 1972; Orvik and Mork 1995). The conflation comes about by using the relation for quasi-Doppler shifts $(U k)$ while examining the connection between a forcing frequency and an observed frequency, on which the quasi-Doppler relation has, in fact, no bearing. Instead, the pertinent theory is that of Doppler shifts.

In a revisit of these interpretations, the first question that arises is whether there really was a "shift" in the first place. In this respect it is of great significance that these studies focused on near-inertial waves rather than on internal tides. Internal tides are forced at precise and well-known frequencies (semidiurnal lunar $M_{2}$ etc.). In contrast, the forcing of near-inertial waves is more blurred. First, because the mechanism primarily responsible for their generation, geostrophic adjustment, generates a range of near-inertial frequencies. Second, because the notion of "inertial" changes, by definition, with latitude. So, if one finds a spectral peak at $f+\delta$ (local Coriolis parameter $f$ ), does this signify a Doppler shift or does it simply result from generation at a higher latitude where the local $f$ was larger? These uncertainties give room for seeing "Doppler shifts" when, in fact, none is there.

For internal tides, on the other hand, the contradiction between the expected "Doppler shifts" (on the basis of 
the quasi-Doppler relation!) and the absence thereof in the moored observations was more patent. To find a way out of this contradiction, one had to resort to rather implausible explanations: Frankignoul (1970) puts forward the idea that the waves come from all directions so that the Doppler effects cancel in the mean; White (1972) assumes that the spectral tidal peak is entirely barotropic, in which case the presumed Doppler shift would be negligible.

These problems disappear at once when one realizes that mean flows cannot create a Doppler shift. Yet, they affect the observations in other ways, as we will now discuss.

\section{a. Near-inertial waves}

For near-inertial waves we can use the dispersion relation (19). (This expression is based on the "Traditional Approximation," which may not always be valid, but for simplicitly it is assumed to hold here.) The dispersion relation with and without a mean flow is shown in Fig. 5. The mean flow brings about two changes. First, for a given frequency the wavenumber is modified. Second, the lower bound of the range of allowable frequencies decreases. The frequency can now attain values lower than $f$. Contrary to what was suggested by Zhai et al. (2005), however, this does not imply a Doppler shift. There is no difference between the emitted and received frequencies for sources and observers that do not move relative to each other, see (21). Figure 5 simply means that waves forced at frequencies slightly below $f$ can now freely propagate away, whereas they could not if no mean flow were present.

All this is not to say that Doppler shifts cannot occur for near-inertial waves. After all, we may be dealing with a moving source if the waves are due to the passage of an atmospheric depression. In a moored instrument, the observed frequency will then be different from the forcing frequency but, since the forcing involves a range of near-inertial frequencies, the actual Doppler shift may be difficult to establish. Another complication arises from the fact that one needs to take into account the angle between the trajectory of the source and the line connecting the source and observer. This aspect goes beyond the scope of this paper but has been dealt with in the literature (e.g., Young 1934).

\section{b. Internal tides}

For internal tides, generated over topography, the source is not moving. If the waves are detected at a fixed mooring, there will be no Doppler shift in frequency, irrespective of the presence of background flows.

Although generation over topography is the typical and most important mechanism for internal tides, an

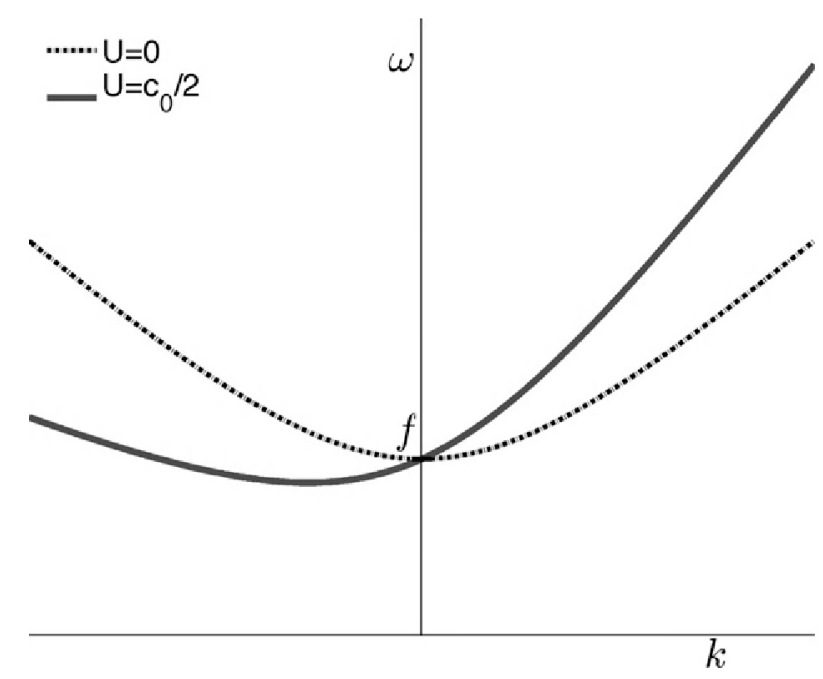

FIG. 5. The dispersion relation (19) plotted for two different cases: without mean flow (dashed), and with mean flow (solid). Notice that in the latter case, $\omega$ attains values lower than $f$.

alternative mechanism can be at work in some configurations, namely when they are produced dynamically by a horizontal displacement of fronts (Ou and Maas 1988). As fronts have sloping isopycnals, horizontal tidal motion pushes isopycnals periodically away from their original position. The situation is complicated since the density gradient associated with the front is normally in geostrophic equilibrium with the Coriolis force acting on a sheared mean flow parallel to the front. But, the tide displaces this geostrophic flow laterally as well, such that over a flat bottom the geostrophic equilibrium is actually retained. To generate internal tides this balance of forces needs to be broken, either by bottom slopes $\mathrm{Ou}$ and Maas 1988) or by instability of the geostrophic front. The complexity of the tidal conversion within a sheared frontal system makes it hard to quantify the particular speed $v_{s}$ at which the source moves. But one can think of, for example, mesoscale variations that lead to a movement of the intersection between the front (which itself oscillates with the barotropic tidal flow) and a topographic feature. The location of that intersection forms the source, which is thus in movement. The crucial point in the present context is that such a movement of the source would create a Doppler shift for fixed observers (a mooring).

This intricate mechanism deserves further study, but the literature already provides an example, from the Straits of Florida, where such a mechanism may be at work. Soloviev et al. (2003) show that in summer, when fronts are strongest, a large and amplified baroclinic spectral peak occurs near periods of about $10 \mathrm{~h}$, while the common semidiurnal peak (still present in the barotropic 
surface tide) is absent. It is not immediately clear that this peak actually represents an internal tide since evidence that one is dealing with one is normally based on its dominant frequency. Perhaps for this reason, while acknowledging the possibility that they may have measured a Doppler-shifted internal tide, Soloviev et al. prefer to interpret this peak as showing the near-resonant response of a local seiche, transverse to the channel between Florida and the Bahamas. Alternatively, they suggest a shift of the "effective" inertial frequency away from the planetary inertial frequency by local subinertial vorticity of about $4 f$.

In the winter season the baroclinic spectral peak, while weaker, does appear at the semidiurnal frequency. Thus, it is plausible that also in summer the semidiurnal internal tide must be there, but, apparently, "blue shifted." This suggestion is reinforced by a seasonal blue shift in the diurnal tide (Soloviev et al. 2003). In view of the previous discussion, apparently the nature of the tidal conversion changes from winter to summer, and we note that instabilities of the Florida Current and nonuniform bathymetry, required to break the geostrophic balance, are indeed present (Davis et al. 2008).

Let us now make a crude estimate of the required source speed, $v_{s}$, based on the observed Doppler-shifted internal tidal frequency. For this, it is important to reiterate that, although mean flows cannot create Doppler shifts, they will modify an existing one; see (3) and (15). For an average Florida Current speed $U \approx 2 \mathrm{~m} \mathrm{~s}^{-1}$, a typical first mode (or interfacial) long wave phase speed $c=O(0.2) \mathrm{m} \mathrm{s}^{-1}$, and a fixed observer $\left(v_{o}=0\right)$, the observed 2 -h period shift of the semidiurnal and diurnal tides suggest $v_{s} \approx U / 5=0.4 \mathrm{~m} \mathrm{~s}^{-1}$. Interestingly, (13) also predicts that, simultaneously with the blue shift, the amplitude of the internal tide generated by a moving source (as compared to that for a source at rest, $v_{s}=0$ ), is boosted by a factor of $5 / 4$, not unlike the summer amplification of the shifted internal tide found on the southeast Florida Shelf (Soloviev et al. 2003).

Thus, the observations offer some support for the speculation that we are here dealing with a Doppler shifted internal tide, due to a moving source. However, we emphasize that this case should nevertheless be considered exceptional for, as mentioned above, internal tide generation occurs predominantly over topography, in which case the source does not move and there will be no Doppler shifts for moored observers.

\section{Conclusions}

The main purpose of this paper is to point out the importance of distinguishing quasi-Doppler shifts (i.e., the difference in frequency between two observers: one fixed and one moving with the mean flow) from Doppler shifts in the normal physical sense, where one compares the frequency of an observer with that of a source.

Regarding the latter, the principal points can be summarized as follows.

(i) A Doppler shift in frequency can occur only if there is a relative movement of source and observer (sections 2 and 3 ).

(ii) The presence of a mean flow does not change this fact (sections 2 and 3).

(iii) The wavelength and amplitude are affected by a mean flow as well as by a movement of the source (section 3).

(iv) No meaningful interpretation of observational data in terms of Doppler shifts can be made unless the movement of the observer (e.g., fixed as in moored instrumentation or moving as in a drifting platform) as well as the movement of the source has been ascertained (Section 5).

(v) For dispersive waves, a straightforward generalization of the classical formula for Doppler shifts can be derived (section 4 ). The aforementioned points all remain valid in that case.

Regarding quasi-Doppler shifts and its central relation (17), which plays a key role in Bretherton and Garrett (1968) and following studies, it should be noted that the movement of a source does not feature in this relation; it is invariant for that movement (section $3 \mathrm{e}$ ). But, this should not be misinterpreted as implying that the movement of a source does not influence the individual terms $\omega, \omega^{\prime}$, and $k$. The invariance just means that (17) cannot tell us anything about that influence; for this, it is necessary to consider Doppler shifts in the strict sense of the word.

In this paper we have restricted ourselves to the relatively straightforward cases in which all velocities are parallel and in which the mean flow is spatially uniform. The more general cases of arbitrary angles between the various velocities and of spatial variations in the mean flow were analyzed by Bateman (1931) and Young (1934). In particular, the mean flow at the location of the source may be different from that at the location of the observer. The upshot is that mean flows still create no Doppler shifts. This is actually already clear from the "stationarity argument" discussed in section 2 .

In reality, mean flows vary not only spatially but also in time. This aspect, however, brings in a new element quite distinct from Doppler shifts, namely nonlinear interaction, the generation of higher harmonics, etc. Such interactions do not bring about a shift in the spectral peak, but rather they create new additional peaks at the sum and difference frequencies of mean flow and wave 
(see, e.g., Bell 1976). If the flow oscillates slowly compared to the wave or if the stratification varies slowly, these peaks will be very close to the main peak, effectively making it broader (depending on the spectral resolution; in the time domain, this is manifested as intermittency). To add to the already existing confusion, this effect is often called "Doppler smearing," even though it has nothing to do with Doppler shifts or, indeed, with anything Christian Doppler ever did.

Acknowledgments. We thank Dirk Olbers and an anonymous referee for their critical comments.

\section{REFERENCES}

Bateman, H., 1917: Döppler's principle for a windy atmosphere. Mon. Wea. Rev., 45, 441-442.

_ 1931: Sound rays as extremals. J. Acoust. Soc. Amer., 2, 468475.

Bell, T. H., 1976: Doppler effects of inertial currents on subsurface temperature measurements. J. Mar. Res., 34, 457-468.

Bretherton, F. P., and C. J. R. Garrett, 1968: Wave trains in inhomogeneous moving media. Proc. Roy. Soc., A302, 529 554.

Bühler, D. R., 2009: Waves and Mean Flows. Cambridge University Press, $341 \mathrm{pp}$.

Davis, K. A., J. J. Leichter, J. L. Hench, and S. G. Monismith, 2008: Effects of western boundary current dynamics on the internal wave field of the Southeast Florida shelf. J. Geophys. Res., 113, C09010, doi:10.1029/2007JC004699.
Doppler, C., 1907: Abhandlungen. H. A. Lorentz, Ed., Engelmann, Leipzig. $195 \mathrm{pp}$.

Frankignoul, C. J., 1970: The effect of weak shear and rotation on internal waves. Tellus, 22, 194-204.

Kunze, E., 1985: Near-inertial wave propagation in geostrophic shear. J. Phys. Oceanogr., 15, 544-565.

Lighthill, J., 1978: Waves in Fluids. Cambridge University Press, $504 \mathrm{pp}$.

Lord Rayleigh, 1896: The Theory of Sound. 2nd ed. Vol. II, MacMillan, 504 pp.

Messiah, A., 1961: Quantum Mechanics. Vol. I, North-Holland Publishing Company, 504 pp.

Olbers, D. J., 1981: The propagation of internal waves in a geostrophic current. J. Phys. Oceanogr., 11, 1224-1233.

Orvik, K. A., and M. Mork, 1995: A case study of Doppler-shifted inertial oscillations in the Norwegian Coastal Current. Cont. Shelf Res., 15, 1369-1379.

Ou, H. W., and L. R. M. Maas, 1988: Tides near a shelf-slope front. Cont. Shelf Res., 8, 729-736.

Soloviev, A. V., M. E. Luther, and R. H. Weisberg, 2003: Energetic baroclinic super-tidal oscillations on the southeast Florida shelf. Geophys. Res. Lett., 30, 1463, doi:10.1029/2002GL 016603.

Toman, K., 1984: Christian Doppler and the Doppler effect. Eos, Trans. Amer. Geophys. Union, 65, 1193-1194.

White, W. B., 1972: Doppler shift in the frequency of inertial waves observed in moored spectra. Deep-Sea Res., 19, 595-600.

Young, R. W., 1934: The Doppler effect for sound in a moving medium. J. Acoust. Soc. Amer., 6, 112-114.

Zauderer, E., 1989: Partial Differential Equations of Applied Mathematics. 2nd ed. Wiley, 891 pp.

Zhai, X., R. J. Greatbach, and J. Sheng, 2005: Doppler-shifted inertial oscillations on a $\beta$ plane. J. Phys. Oceanogr., 35, 1480-1488. 